\title{
FREQUENCY SHIFT OF THE PROPAGATING ULTRAINTENSE FIELD IN A PLASMA WITH A FRACTION OF ELECTRON-POSITRON PAIRS UNDER THE CONDITIONS OF RESPONSE SATURATION
}

\author{
O.B. Shiryaev
}

\section{General Physics Institute RAS, Moscow, Russia}

Currently obtainable laser intensities being high enough to trigger the production of electron-positron pairs in laser-driven plasmas via the scattering of fast electrons off the nuclei, it is of interest to examine how the resulting infusion of a sparse fraction of electron-positron pairs into an ion-electron plasma would modify its response to strong external electromagnetic field and, as the next step, how the modification would factor into the picture of the nonlinear propagation. The interactions between an intense electromagnetic wave and a plasma consisting of ions, electrons, and a minority population of electron-positron pairs are studied in the present paper using a self-consistent 3D model which is derived from the Maxwell and relativistic fluid dynamics equations. The assumptions built into the model are that the propagating field is an intense circularly polarized envelope which is also sufficiently short to consider the ions immobile and that the plasma is cold, underdense, and unmagnetized. An exact solution to the corresponding set of equations is presented to describe a plane wave of arbitrarily high intensity propagating into the composite plasma and exhibiting a generally intensity-dependent frequency shift which is determined by the parameters of the nonlinear interaction. Analysis of the part of the solution accounting for the plasma behavior in the driving field shows that, at intensities exceeding the relativistic threshold by a factor of several, the electron and positron fluids switch to a saturated mode, with the plasma response depending weakly on the incident wave strength from within the ultrarelativistic range. Importantly, the saturation is reached even for the concentrations of the pair fraction far below the background ion and electron concentrations. An expansion applicable in the ultrarelativistic incident intensity limit is suggested to express the plasma wake via the driving field strength and the concentration of the electron-positron pairs, and, within its scope, the above plane wave proves to be stable.

A relation between the frequency shift and the concentration of pairs in the strongfield case is established. It is demonstrated that the frequency shift induced by the interaction with a plasma containing pairs differs essentially from the one found under the reference scenario of the propagation of an intense electromagnetic wave into a plasma without the admixture of pairs. Potentially, the finding can be useful in identifying the process of pair creation in laser-driven plasmas.

1. O.B. Shiryaev, Phys Plasmas 20, 52112 (2013) 